\title{
The Last Three Minutes: Issues in Gravitational-Wave Measurements of Coalescing Compact Binaries
}

\author{
Curt Cutler, ${ }^{(1)}$ Theocharis A. Apostolatos, ${ }^{(1)}$ Lars Bildsten, ${ }^{(1)}$ Lee Samuel Finn, ${ }^{(2)}$ Eanna E. Flanagan, ${ }^{(1)}$ \\ Daniel Kennefick, ${ }^{(1)}$ Dragoljub M. Markovic, ${ }^{(1)}$ Amos Ori, ${ }^{(1)}$ Eric Poisson, ${ }^{(1)}$ Gerald Jay Sussman, ${ }^{(1)}$,(a) \\ and Kip S. Thorne ${ }^{(1)}$ \\ (1) Theoretical Astrophysics, California Institute of Technology, Pasadena, California 91125 \\ ${ }^{(2)}$ Department of Physics and Astronomy, Northwestern University, Evanston, Illinois 60208
}

(Received 24 August 1992)

\begin{abstract}
Gravitational-wave interferometers are expected to monitor the last three minutes of inspiral and final coalescence of neutron star and black hole binaries at distances approaching cosmological, where the event rate may be many per year. Because the binary's accumulated orbital phase can be measured to a fractional accuracy $\ll 10^{-3}$ and relativistic effects are large, the wave forms will be far more complex and carry more information than has been expected. Improved wave form modeling is needed as a foundation for extracting the waves' information, but is not necessary for wave detection.
\end{abstract}

PACS numbers: 04.30.+x, 04.80.+z, 97.60.Jd, 97.60.Lf

A network of gravitational-wave interferometers (the American LIGO [1], the French/Italian VIRGO [2], and possibly others) is expected to be operating by the end of the 1990s. The most promising waves for this network are from the inspiral and coalescence of neutron star (NS) and black hole $(\mathrm{BH})$ binaries $[3,4]$, with an estimated event rate of $\sim(3 /$ year $)[\text { distance } /(200 \mathrm{Mpc})]^{3}[5]$. This Letter reports initial results of a new research effort that is changing our understanding of these waves; further details will be given in the authors' papers cited in the references.

A binary's inspiral and coalescence will produce two gravitational wave forms, one for each polarization. By cross correlating the outputs of three or more interferometers, both wave forms can be monitored and the source's direction can be determined to within $\sim 1$ degree $[4,6]$.

We shall divide each wave form into two parts: the inspiral wave form, emitted before tidal distortions become noticeable $(\lesssim 10$ cycles before complete disruption or merger $[7,8]$ ), and the coalescence wave form, emitted during distortion, disruption, and/or merger.

As the binary, driven by gravitational radiation reaction, spirals together, its inspiral wave form sweeps upward in frequency $f$ (it "chirps"). The interferometers will observe the last several thousand cycles of inspiral (from $f \sim 10 \mathrm{~Hz}$ to $\sim 1000 \mathrm{~Hz}$ ), followed by coalescence.

Theoretical calculations of the wave forms are generally made using the post-Newtonian (PN) approximation to general relativity. Previous calculations have focused on the Newtonian-order wave forms $[1,3,4,9]$ and on PN modulations of their amplitude and frequency [10].

We have recently realized that the $\mathrm{PN}$ modulations are far less important than PN contributions to the secular growth of the waves' phase $\Phi=2 \pi \int f d t$, which arise largely from $\mathrm{PN}$ corrections to radiation reaction $[11,12]$. The binary's parameters are determined by integrating the observed (noisy) signal against theoretical templates, and if the signal and template lose phase with each other by as little as one half cycle over the thousands observed as the signal sweeps through the interferometers' band, their overlap integral will be strongly reduced. This sensitivity to phase does not mean that accurate templates are needed in searches for the waves (see below). However, once the waves have been found, and if accurate templates are in hand, then from the orbital phasing one can infer each of the system's parameters $\lambda_{i}$ to an accuracy of order the change $\Delta \lambda_{i}$ which alters by unity the number of cycles $\mathcal{N}_{\text {cyc }}$ spent in the interferometers' band.

We shall assume (as almost always is the case) that the binary's orbit has been circularized by radiation reaction [10]. Then the only parameters $\lambda_{i}$ that can significantly influence the inspiral template's phasing are the bodies' masses, vectorial spin angular momenta, and spin-induced quadrupole moments (which we shall ignore because, even for huge spins, they produce orbital phase shifts no larger than $\sim 1$ [8]). More specifically, the number of cycles spent in a logarithmic interval of frequency, $d \mathcal{N}_{\text {cyc }} / d \ln f=(1 / 2 \pi)(d \Phi / d \ln f)$, is

$$
\begin{aligned}
\frac{d \mathcal{N}_{\text {cyc }}}{d \ln f}= & \frac{5}{96 \pi} \frac{1}{\mu M^{2 / 3}(\pi f)^{5 / 3}}\left\{1+\left(\frac{743}{336}+\frac{11}{4} \frac{\mu}{M}\right) x\right. \\
& \left.-[4 \pi+\text { S.O. }] x^{1.5}+[\text { S.S. }] x^{2}+O\left(x^{2.5}\right)\right\} .
\end{aligned}
$$

Here $M$ is the binary's total mass, $\mu$ its reduced mass, and $x \equiv(\pi M f)^{2 / 3} \simeq M / D$ the PN expansion parameter (with $D$ the bodies' separation and $c=G=1$ ). The PN correction $\left[O(x)\right.$ term] is from [13]. In the $\mathrm{P}^{1.5} \mathrm{~N}$ correction $\left[O\left(x^{1.5}\right)\right.$ term], the $4 \pi$ is created by the waves' interaction with the binary's monopolar gravitational field as they propagate from the near zone to the radiation zone [14], and the "S.O." denotes contributions due to spin-orbit coupling [15]. In the $\mathrm{P}^{2} \mathrm{~N}$ correction the "S.S." includes spin-spin coupling effects [15] plus an expression quadratic in $\mu / M$. (For bodies with sizes comparable to their separations, the spin-orbit and spin-spin terms are of $\mathrm{PN}$ order; but the compactness of a $\mathrm{BH}$ or NS boosts them up to $\mathrm{P}^{1.5} \mathrm{~N}$ and $\mathrm{P}^{2} \mathrm{~N}$, respectively; cf. [15].)

Since the leading-order, Newtonian contribution to 
Eq. (1) gives several thousand cycles in the interferometers' band, to be measurable the higher-order corrections need only be as large as a part in several thousand, even when the signal is only a little above the threshold for detection. It is this that gives measurements based on the waves' phasing their high potential accuracy.

The determination of the binary's masses and spins is made possible by the various frequency dependences in Eq. (1). Analytic [11, 12] and Monte Carlo [11] calculations show that (i) the chirp mass $M_{c} \equiv \mu^{3 / 5} M^{2 / 5}$ [which governs the Newtonian part of (1)] will typically be measured to a few tenths of a percent, and (ii) if we somehow knew that the spins were small, then the reduced mass $\mu$ would be measured to $\sim 1 \%$ for NS-NS and NS-BH binaries, and $\sim 3 \%$ for $\mathrm{BH}-\mathrm{BH}$ binaries. (Here and below $\mathrm{NS}$ means $\mathrm{a} \sim 1.4 M_{\odot}$ neutron star and $\mathrm{BH}$ means $\mathrm{a} \sim 10 M_{\odot}$ black hole.) These accuracies assume a noise spectrum whose shape is that of "advanced detectors" [1], and the use of two detectors with amplitude signalto-noise ratios of 9 in each. Unfortunately, the frequency (x) dependences of the various terms in Eq. (1) are not sufficiently different to give a clean separation between $\mu$ and the spins. Preliminary estimates [11] in which the S.O. term in Eq. (1) was taken into account but not the S.S. term, suggest that the spin $/ \mu$ correlation will worsen the typical accuracy of $\mu$ by a factor $\sim 15$, to $\sim 10 \%$ for NS-NS, $\sim 15 \%$ for NS-BH, and $\sim 70 \%$ for $\mathrm{BH}-\mathrm{BH}$. These worsened accuracies might be improved significantly by wave form modulations caused by the spin-induced precession of the orbit [16] (see Fig. 1), and even without modulational information, a certain combination of $\mu$ and the spins will be determined to a few percent. Much additional theoretical work is needed to firm up the measurement accuracies.

Although the early, $10 \mathrm{~Hz}-50 \mathrm{~Hz}$ part of the wave form produces only $\sim 10 \%$ of the signal-to-noise squared, it accounts for $>80 \%$ of the measurable cycles and most of the mass-measurement accuracy. It thus is important that the interferometers achieve good low-frequency performance.

From the above accuracies, we estimate that, when searching for inspiral signals in noisy data, one will need $\sim 10^{5}$ templates to cover the possible values of masses and spins. Since two otherwise identical wave forms displaced by $3 \mathrm{~ms}$ will have their overlap integral reduced substantially, $\sim 10^{15}$ templates per year must be integrated against the data. The noise is expected to be Gaussian (thanks to filtering with the templates and cross correlation of detectors), and thus the probability that any particular template will produce a spurious signal-to-noise ratio $S / N>\rho$ in each of two detectors is $[\operatorname{erfc}(\rho / \sqrt{2})]^{2}$. For $\rho=(5,6,7), \quad[\operatorname{erfc}(\rho / \sqrt{2})]^{2} \approx$ $\left(10^{-13}, 10^{-18}, 10^{-23}\right)$. Thus an appropriate threshold for detection is $S / N \geq 6.0$. Notice that this threshold is quite insensitive to our estimate of $\sim 10^{15}$ templates/year, and it was increased by only $10 \%$ (from 5.5 to 6.0 ) by our discovery of the rich influence of $\mu$ and

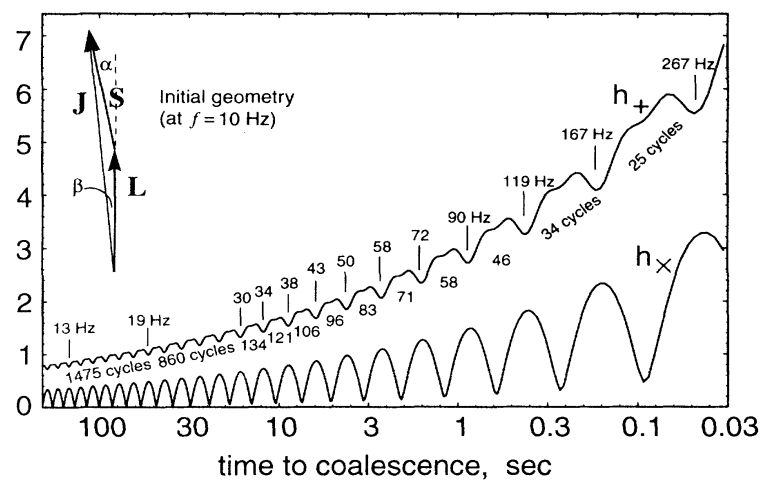

FIG. 1. This figure illustrates the importance of spininduced orbital precession (the "dragging of inertial frames"), which has been ignored in all previous discussions of gravitational waves from inspiraling binaries. Here a $1 M_{\odot}$ nonspinning NS spirals into a $10 M_{\odot}$, rapidly spinning Kerr black hole (spin parameter $a=1$ ). The orbital angular momentum $\mathbf{L}$ is inclined by $\alpha=11.3$ degrees to the hole's spin angular momentum $\mathbf{S}$, and the two precess around $\mathbf{J}=\mathbf{L}+\mathbf{S}$, whose direction remains fixed in space as $L=|\mathbf{L}|$ shrinks and $S=$ $|\mathbf{S}|=M_{\mathrm{BH}}^{2} a$ remains constant $\left(L / S=(\mu / M a)(\pi M f)^{-1 / 3} \simeq\right.$ $\left.[(5 \mathrm{~Hz}) / f]^{1 / 3} \lesssim 1\right)$. The precession of $\mathbf{L}$ around $\mathbf{J}$, with precession angle $\beta \simeq \alpha /(1+L / S) \simeq 0.11$ to 0.17 , modulates the waves by $\delta h \sim(0$ to 4$) \beta \times\left[\max \left(h_{+}, h_{\times}\right)\right]$, where the coefficient depends on (i) the direction to Earth (here out of the paper) and (ii) the orientation of the detector's arms (here horizontal and vertical for $h_{+}$, and rotated 45 degrees for $h_{\times}$). The figure shows the wave forms' modulational envelopes (in arbitrary units, the same for $h_{+}$and $h_{\times}$), parametrized by the wave frequency $f$ and the number of cycles of oscillation between the indicated $f$ 's. The total number of precessions from $f$ to coalescence is $N_{\text {prec }} \simeq(5 / 64 \pi)(M a / \mu)(\pi M f)^{-2 / 3} \simeq$ $20[f /(10 \mathrm{~Hz})]^{-2 / 3}$. For further details see Ref. [16].

spins on the orbital phase. For "advanced-detector sensitivities" [1], NS-NS inspirals will exceed this threshold at distances $\lesssim 1 \mathrm{Gpc}$, and $\mathrm{BH}-\mathrm{BH}$ inspirals at $\lesssim 5 \mathrm{Gpc}$.

We suspect that, when searching for inspiral signals, it will be sufficient to use templates accurate to $\mathrm{P}^{1.5} \mathrm{~N}$ order; the omitted, higher-order corrections will be compensated by incorrect estimates of the masses and spins, and the signals will still be found. Better yet will be a set of templates that span the range of expected wave form behaviors in such a way as to minimize the computational time. Such templates need to be developed.

Although highly accurate wave form templates will not be needed when searching for waves, they will be needed when extracting the waves' information. Making optimal use of the interferometers' data will require generalrelativity-based wave form templates whose phasing is correct to within a half cycle or so during the entire frequency sweep from $\sim 10 \mathrm{~Hz}$ to $\sim 1000 \mathrm{~Hz}$. We estimate that $\mathrm{P}^{1.5} \mathrm{~N}$-order templates (the most accurate computed to date) will produce systematic errors in $\mu$ of order $10 \%$.

By examining an idealized limit $(\mu \ll M$ and vanishing spins), we have found that the PN expansion converges very slowly $[14,17]$. We calculated the waves from such 
a binary to high accuracy using the Teukolsky-ReggeWheeler [18] black-hole perturbation formalism and then fitting a PN expansion to the results, to obtain [17]

$$
\begin{aligned}
& \frac{d \mathcal{N}_{\mathrm{cyc}}}{d \ln f}=\frac{f^{2} d E / d f}{d E / d t}=\frac{5}{96 \pi} \frac{M / \mu}{x^{2.5}} \\
& \quad \times \frac{1-\frac{3}{2} x-\frac{81}{8} x^{2}-\frac{675}{16} x^{3}+\cdots}{1-\frac{1247}{336} x+4 \pi x^{1.5}-4.9 x^{2}-38 x^{2.5}+135 x^{3}+\cdots} .
\end{aligned}
$$

Here $d E / d f$ is half the ratio of the changes of orbital energy $d E$ and orbital frequency $d(f / 2)$ as the orbit shrinks, and $d E / d t$ is the power carried off by gravitational waves. The coefficients in the denominator after the $4 \pi$ were obtained from the fit; all other coefficients are known analytically. The coefficient 4.9 has accuracy $\sim 2 \%$; the 38 , $\sim 10 \%$; and the 135, 50\%.

We can use Eq. (3) to estimate how the phase accuracy depends on the order to which the $\mathrm{PN}$ expansion is carried. Compare a template constructed using Eq. (3) with a $\mathrm{P}^{2} \mathrm{~N}$-order template [all $x^{2.5}$ and $x^{3}$ terms in the denominator of Eq. (3) omitted]. Adjust the constants of integration so the templates are perfectly in phase at $70 \mathrm{~Hz}$, where the detector has maximum sensitivity [1]. Then the two templates fall out of phase as $f$ decreases below $70 \mathrm{~Hz}$ and increases above $70 \mathrm{~Hz}$; for a NS-BH binary the phase difference has grown to half a cycle at $40 \mathrm{~Hz}$ and $140 \mathrm{~Hz}$, and to three cycles at $10 \mathrm{~Hz}$ and 400 $\mathrm{Hz}$. If the template were computed through $\mathrm{P}^{2.5} \mathrm{~N}$ order, there would be no substantial improvement. It is not at all clear how far beyond $\mathrm{P}^{2.5} \mathrm{~N}$ the template must be carried to keep its total phase error below a half cycle over the entire range from $\sim 10 \mathrm{~Hz}$ to $\sim 400 \mathrm{~Hz}$, but the slowness of the convergence suggests that new techniques are needed for computing templates to higher accuracies.

One possible new technique is a "weak-reaction expansion." This would be a variant of numerical relativity in which one expands in powers of $1 / Q \equiv(d E / d t)(\pi f E)^{-1}$ (a measure of the effects of radiation reaction during one orbit). Because $1 / Q \sim x^{2.5}$ (with $x$ the $\mathrm{PN}$ parameter) is always $\ll 1$, this expansion might converge quickly. The expansion might begin with a Blackburn-Detweiler [19] type numerical solution of the Einstein equations with standing-wave boundary conditions. From the standing waves, one can read off the gravitational radiation that results when one switches to outgoing waves, and thereby infer the wave forms and inspiral rate to first order in $1 / Q$. It is an important challenge to devise a way to iterate to second order.

We turn now from a binary's inspiral wave forms to its coalescence wave forms. By the beginning of coalescence, the binary's parameters (masses, spins, geometry) will be known with fair accuracy, and from its masses the nature of its bodies (BH versus NS) should be fairly clear. The coalescence wave forms can then be used to probe issues in the physics of gravity and atomic nuclei. For $\mathrm{BH}-\mathrm{BH}$ binaries, the issue to be probed (large-amplitude, highly nonlinear vibrations of spacetime curvature) has been much discussed; see, e.g., [1] and references therein. Not previously discussed, however, is the possibility of using NS-BH and NS-NS coalescence to measure the NS mass-radius relation (from which one can infer the equation of state of nuclear matter [20]):

For a NS-BH binary with the $\mathrm{BH}$ spinning moderately fast, the NS should disrupt tidally before plunging into the BH. The NS disruption should be quick, as should the final coalescence of a NS-NS binary: within several orbits the binary should get smeared into a roughly axially symmetric configuration, thereby shutting off its waves $[7,8$, 21]. The waves' inspiral frequency at the beginning of tidal disruption will be $f_{\mathrm{td}}=(\alpha / 2 \sqrt{2} \pi) \sqrt{M_{\mathrm{NS}} / R_{\mathrm{NS}}^{3}} \simeq$ $(1.5 \mathrm{kHz}) \sqrt{\left(M_{\mathrm{NS}} / 1.4 M_{\odot}\right)\left[(10 \mathrm{~km}) / R_{\mathrm{NS}}\right]^{3}}$, where $M_{\mathrm{NS}}$ and $R_{\mathrm{NS}}$ are the mass and radius of the less massive (or only) neutron star, and $\alpha$ is a factor close to 1 that will depend only weakly on the mass and spin of the companion. Since $M_{\mathrm{NS}}$ will be known from the earlier inspiral waves, a measurement of $f_{\mathrm{td}}$ will reveal $R_{\mathrm{NS}}$.

Shot noise may prevent the LIGO and VIRGO "workhorse," broad-band interferometers from measuring $f_{\mathrm{td}}$; but special, narrow-band, "dual recycled" interferometers [22] may do the job. Such an interferometer could give a simple "yes or no" answer as to whether the waves swept through its frequency band $f_{0} \pm \Delta f$ (and if yes, the spectral density of wave energy in that band), with an amplitude signal-to-noise ratio [23]

$$
\frac{S}{N} \simeq 8 \frac{\left[\eta I_{0} /(100 \mathrm{~W})\right]^{1 / 2}}{\left(A^{2} / 10^{-5}\right)^{1 / 2}} \frac{\left(\mu / 1 M_{\odot}\right)^{1 / 2}\left(M / 10 M_{\odot}\right)^{1 / 3}}{[r /(200 \mathrm{Mpc})]\left[f_{0} /(1 \mathrm{kHz})\right]^{7 / 6}}
$$

Here $\eta$ is the photodiode efficiency, $I_{0}$ the laser power, and $A^{2}$ the fractional light power lost to absorption and scattering in each bounce off a mirror. By collecting such data on a number of binaries, with various choices for the interferometer's frequency $f_{0}$, it should be possible to zero in on $f_{\mathrm{td}}$ for various types of binaries, and thence on the NS radius-mass relation.

As a foundation for these measurements, theorists should model tidal disruption in NS-BH binaries and the coalescence of NS-NS binaries, to determine the dependence of $f_{\mathrm{td}}$ on $R_{\mathrm{NS}}$ and the binary's other parameters, and the shape of the waves' spectrum above $f_{\mathrm{td}}$. (Such modeling is also driven by the possibility that these coalescences produce observed gamma-ray bursts [24].)

We turn next from a binary's coalescence to the use of its wave forms as cosmological probes, via a variant of a method conceived by Schutz [4]: For a binary at a redshift $z \gtrsim 1$, the inspiral wave forms will be measured as functions of redshifted time, and correspondingly they will depend on and reveal the "redshifted masses" $(1+z) M_{1}$ and $(1+z) M_{2}$ and the binary's "luminosity distance" $r_{L}$. From these, one can infer whether the binary contains a NS. Assuming (as observations suggest) that NS masses in binaries cluster strongly around $1.4 M_{\odot}$, 
one can estimate $1+z$ as the observed $M_{\mathrm{NS}}$ divided by $1.4 M_{\odot}$. From a large number of such measured $(1+z)$ 's and $r_{L}$ 's, one can deduce the Universe's redshift-distance relation and thence its Hubble constant $H_{0}=h_{0} \times 100$ $\mathrm{km} \mathrm{s}^{-1} \mathrm{Mpc}^{-1}$, density parameter $\Omega_{0}=(8 \pi / 3)\left(\rho_{0} / H_{0}^{2}\right)$, and cosmological constant $\Lambda=3 H_{0}^{2} \lambda_{0}$.

We have carried out simulations of such a determination of cosmological parameters [25], assuming (i) the LIGO-VIRGO network with three identical interferometers whose noise spectra have the "advanced detector" shape of Ref. [1], (ii) distance errors $\Delta r_{L} / r_{L} \simeq(2 / \rho) \times($ a function, $\geq 1$, of the binary direction and orientation), and (iii) mass errors $\Delta M_{\mathrm{NS}} / M_{\mathrm{NS}} \simeq 1.4 / \rho$; here $\rho$ is the amplitude signal-to-noise ratio. By contrast with electromagnetic cosmological measurements, which suffer from light absorption and source evolution, this method will suffer just one type of propagation noise (gravitational lensing by mass inhomogeneities), and probably no evolutionary effects (it seems unlikely that the NS mass spectrum will depend on cosmological epoch).

Our simulations [25] show that gravitational lens noise should be negligible compared to detector noise, and they suggest the following one-sigma errors in the cosmological parameters inferred from NS-BH binary measurements. (i) $\Delta h_{0} \simeq 0.01 N(\hat{\tau} \mathcal{R})^{-1 / 2}$ for $N \lesssim 3$, where $N$ is the detectors' noise (strain $/ \sqrt{\mathrm{Hz}}$ ) in units of the "advanced detector" level shown in Ref. [1], $\mathcal{R}$ is the event rate in units of the best estimate, 100 $\mathrm{yr}^{-1} \mathrm{Gpc}^{-3}$, and $\hat{\tau}$ is the observation time in years. (ii) In a "compact" universe $\left(h_{0}=1, \Omega_{0}=1, \lambda_{0}=0\right)$, $\Delta \Omega_{0} \simeq 0.1 N^{2}(\hat{\tau} \mathcal{R})^{-1 / 2}$ and $\Delta \lambda_{0} \simeq 0.2 N^{1.5}(\hat{\tau} \mathcal{R})^{-1 / 2}$ for $N \lesssim 1$. (iii) In a "spacious" universe $\left(h_{0}=0.5, \Omega_{0}=0.2\right.$, $\left.\lambda_{0}=0\right), \Delta \Omega_{0} \simeq N^{3}(\hat{\tau} \mathcal{R})^{-1 / 2}$ and $\Delta \lambda_{0} \simeq N^{2.5}(\hat{\tau} \mathcal{R})^{-1 / 2}$ for $N \lesssim 0.75$. For measurements based on NS-NS binaries rather than NS-BH, the fact that one can use the highly accurate chirp mass to deduce the redshift instead of the much less accurate $M_{\mathrm{NS}}$ does not significantly compensate for the weaker NS-NS signal strength; consequently, the NS-NS-based errors are approximately as quoted above for NS-BH, but with $N$ replaced by $2 N$.

These accuracies suggest that, if event rates are as currently estimated, interesting cosmological measurements, beyond determining $h_{0}$, cannot begin until the detector sensitivities reach the "advanced detector level."

Some of the issues discussed above have implications for a possible future space-based interferometer LAGOS, which would operate in the band $0.0001-0.03 \mathrm{~Hz}$ [26].

For helpful discussions we thank D. Chernoff, L. Kidder, A. Krolak, E. S. Phinney, B. F. Schutz, C. M. Will, and A. Wiseman. This research was supported in part by NSF Grant No. PHY-9213508, and, in view of its applications to LAGOS, by NASA Grants No. NAGW2897, No. 2920, and No. 2936, and by a Lee A. Dubridge Fellowship to L. Bildsten, an Alfred P. Sloan Foundation Fellowship to L. S. Finn, and a NSERC Fellowship to E. Poisson. (a) Permanent address: Dept. of Electrical Engineering, MIT, Cambridge, MA 02139.

[1] A. Abramovici et al., Science 256, 325 (1992).

[2] C. Bradaschia et. al., Nucl. Instrum. Methods Phys. Res., Sect. A 289, 518 (1990).

[3] K. S. Thorne, in 300 Years of Gravitation, edited by S. W. Hawking and W. Israel (Cambridge Univ. Press, Cambridge, 1987), p. 330.

[4] B. F. Schutz, Nature (London) 323, 310 (1986); B. F. Schutz, Classical Quantum Gravity 6, 1761 (1989).

[5] R. Narayan, T. Piran, and A. Shemi, Astrophys. J. 379, L17 (1991); E. S. Phinney Astrophys. J. 380, L17 (1991).

[6] Y. Gursel and M. Tinto, Phys. Rev. D 40, 3884 (1990); P. Jaranowski and A. Krolak (unpublished).

[7] C. Kochanek, Astrophys. J. 398, 234 (1992).

[8] L. Bildsten and C. Cutler, Astrophys. J. 400, 175 (1992).

[9] H. D. Wahlquist, Gen. Relativ. Gravitation 19, 1101 (1987)

[10] C. W. Lincoln and C. M. Will, Phys. Rev. D 42, 1123 (1990).

[11] C. Cutler and E. E. Flanagan (unpublished).

[12] L. S. Finn and D. Chernoff, Phys. Rev. D 47, 2198 (1993).

[13] R. V. Wagoner and C. M. Will, Astrophys. J. 210, 764 (1976); 215, 984 (1977).

[14] E. Poisson, Phys. Rev. D 47, 1497 (1993).

[15] L. E. Kidder, C. M. Will, and A. G. Wiseman, Phys. Rev. D (to be published).

[16] G. J. Sussman, T. A. Apostolatos, C. Cutler, and K. S. Thorne (unpublished).

[17] C. Cutler, L. S. Finn, E. Poisson, and G. J. Sussman, Phys. Rev. D 47, 1511 (1993).

[18] S. A. Teukolsky, Astrophys. J. 185, 635 (1973); T. Regge and J. A. Wheeler, Phys. Rev. 108, 1063 (1957).

[19] J. K. Blackburn and S. L. Detweiler, Phys. Rev. D 46, 2318 (1992), but perhaps with their action principle replaced by a relaxation solution of the elliptic equations.

[20] L. Lindblom, Astrophys. J. 398, 569 (1992).

[21] F. A. Rasio and S. L. Shapiro, Astrophys. J. 401, 226 (1992).

[22] A. Krolak, J. A. Lobo, and B. J. Meers Phys. Rev. D 43, 2470 (1991).

[23] $S / N=\left(\pi \mu M^{3} / 5 r^{2}\right)^{1 / 2}\left(\pi M f_{0}\right)^{-7 / 6} \mathcal{H}_{2}\left[\int_{0}^{\infty} d f / S_{h}(f)\right]^{1 / 2}$, from Ref. [3] Eqs. (30) [with 3 replaced by 6 due to an error in (29)], (44) [with 12 replaced by 6 due to $f t$ being corrected to $\int f d t$ in (42)], and (110); $\mathcal{H}_{2} \simeq 0.8$ is a general relativistic correction for the NS-BH case [L. S. Finn, A. Ori, and K. S. Thorne (unpublished)]. For $\int_{0}^{\infty} d f / S_{h}(f)$ we use $\left(\pi \eta I_{0} / 2 \hbar\right)(l / \lambda)\left(1 / A^{2}\right)$, where $l=4$ $\mathrm{km}$ is the interferometer arm length and $\lambda=0.4 \mu \mathrm{m}$ is the laser light wavelength [Eq. (3.7) of Ref. [22]] in the regime $c A^{2} / l \ll \Delta f \ll f_{0}$, with $\tau=2 l / c$ and corrections of a multiplicative factor $16 / A^{2}$ and in the denominator $\pi \tau \Delta f \rightarrow 2 \pi \tau \Delta f$; A. Krolak (private communication).

[24] B. Paczynski, Astrophys. J. Lett. 308, L43 (1986); J. Goodman, Astrophys. J. Lett. 308, L47 (1986); D. Eichler, M. Livio, T. Piran, and D. N. Schramm, Nature (London) 340, 126 (1989).

[25] D. M. Markovic (to be published).

[26] J. E. Faller, P. L. Bender, J. L. Hall, D. Hils, R. T. Stebbins, and M. A. Vincent, Adv. Space Res. (COSPAR) 9, 107 (1989). 